\title{
Hardware Testing of the BaBar Drift Chamber Electronics Upgrade
}

\author{
Bryce Littlejohn ${ }^{1}$, Yiwen $\mathrm{Chu}^{2}$, Liv Wiik ${ }^{3}$
}

Office of Science, SULI Program

1) Principia College, 2) Massachusetts Institute of Technology,

\author{
3) Universität Hamburg \\ Stanford Linear Accelerator Center \\ Menlo Park, California
}

August 4, 2005

Prepared in partial fulfillment of the requirements of the Office of Science, U.S. Department of Energy Science Undergraduate Laboratory Internship (SULI) Program under the direction of Jochen Dingfelder and Mike Kelsey in Experimental Group C at the Stanford Linear Accelerator Center (SLAC).

Participants:

Signatures

Research Advisor:

Signature 


\section{Contents}

1 Abstract 3

2 Introduction $\quad 4$

$\begin{array}{llr}3 & \text { Methods and Materials } & 8\end{array}$

3.1 Overview ....................... 8

3.2 Component Test Stand . . . . . . . . . . . . . . . 9

3.3 Calibration Test Stand . . . . . . . . . . . . . . . 11

3.4 DCT Test Stand . . . . . . . . . . . . . . . . . . . 12

3.5 Test Records . . . . . . . . . . . . . . . . . . . 13

4 Results and Discussion $\quad 14$

5 Conclusion $\quad 19$

6 Acknowledgements $\quad 20$ 


\section{Abstract}

Hardware Testing of the BaBar Drift Chamber. BRYCE LITTLEJOHN(Principia College, Elsah, IL 62028) YIWEN CHU(Massachusetts Institute of Technology, Cambridge, MA 02139) LIV WIIK(Universität Hamburg, Germany) JOCHEN DINGFELDER AND MIKE KELSEY(Stanford Linear Accelerator Center, Stanford, CA 94025).

The BaBar drift chamber provides position, timing, and $\mathrm{dE} / \mathrm{dx}$ measurements for charged decay products of the $\Upsilon(4 S)$ resonance at $10.58 \mathrm{GeV}$. Increasing data collection rates stemming from higher PEP II luminosities and background have highlighted dead time problems in the drift chamber's data aquisition system. A proposed upgrade, called Phase II, aims to solve the problem with the introduction of rewritable, higher-memory firmware in the DAQ front-end electronics that lowers dataflow through the system. After fabrication, the new electronics components were tested to ensure proper function and reliability before installation in the detector. Some tests checked for successful operation of individual components, while others operated entire sections of the upgraded system in a mockup drift chamber environment. This paper explains the testing process and presents results regarding performance of the upgrade electronics. 


\section{Introduction}

The BaBar detector makes precision measurements of rare $B, D$, and $\tau$ decays by collecting large amounts of collisional data at the $\Upsilon(4 S)$ resonance. An efficient data acquisition system is essential if approximately 3000 events per second are to be successfully collected. Thus, an important criterion in BaBar detector design is minimization of dead time. Despite a previous upgrade, referred to as Phase I, the drift chamber (DCH) has consistently been the experiment's largest dead time contributor. At the current beam luminosity of $8 \times 10^{33} \mathrm{~cm}^{-2} \mathrm{~s}^{-1}$, average dead times peak at $10 \%$ over short periods, but are generally closer to $2 \%$ [1]. However, as PEP II upgrades bring about increases in luminosity and potentially background, dead time has become a noticeably limiting factor in detector efficiency, and will continue to increase until further changes are implemented.

In the drift chamber, each of 7104 sense wires is surrounded by six field wires that create a logarithmically decreasing electric field centered roughly radially about the sense wire [2]. Incident charged particles ionize fill gas inside the drift chamber. The produced electrons drift towards the sense wire, causing secondary ionizations at small distances from the sense wire as electrons are accelerated by the strong electric field. Continued ionization creates a charge avalanche that induces an electric pulse in the sense wire. This signal, along with inherent background, is fed from the sense wires to sixteen azimuthally symmetric groups of three Front End Assemblies (FEAs). FEA 1, the radially innermost assembly, holds two Front End Elements (FEEs), while FEA 2 and FEA 3 contain one each. The FEAs extract timing and charge information from pulses and prepare accepted events for further selection and storage. The large amount of data generated in the FEA can be regarded as one of the causes of dead time in the detector. 


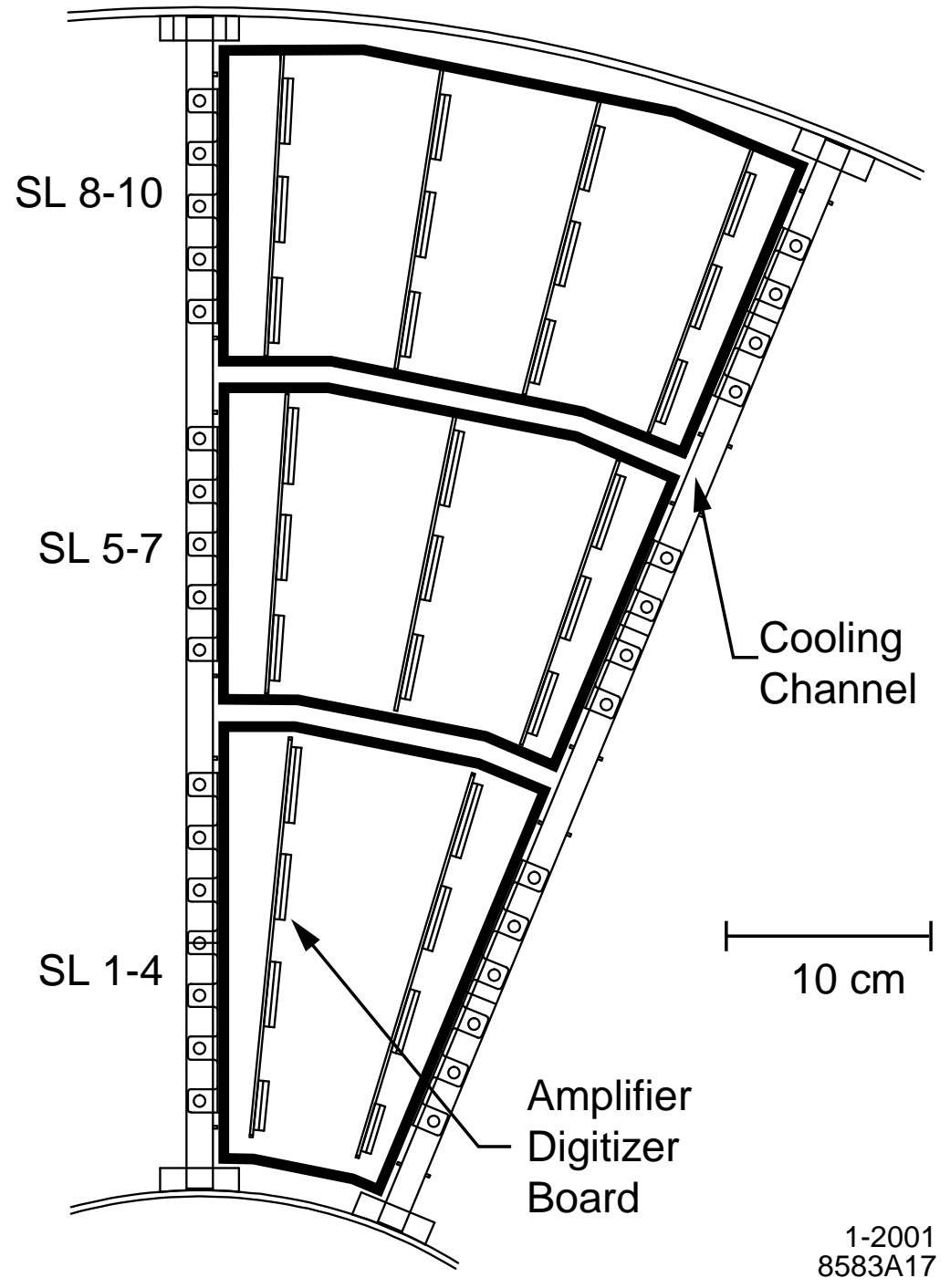

Figure 1: An end-on view of 1/16 of the front-end electronics. The numbers of ADBs per FEA and ELEFANTs per ADB are clearly visible. Taken from [2]. 
The FEAs, shown in Figure 1, are housed in aluminum boxes of three different sizes that conform to the DCH endplate's circular geometry. In the FEAs, an analog signal is first sent to an amplifier-digitizer board (ADB), where it is amplified and transferred to an ELEFANT (ELEctronics For Amplitude aNd Timing) IC. In the ELEFANT, the pulse can be digitized by a Flash Analog-to-Digital Converter (FADC) and marked at its leading edge with a timestamp by a Time-to-Digital Converter (TDC). Created data are then stored in a 12.9 microsecond buffer. Another continuous stream of raw data is concurrently sent through the FEA and provides the necessary information to decide when an acceptable event (Level 1 Accept) has occurred. Once triggered, a readout interface board (ROIB) accepts data from the ELEFANT buffers and sends them to a data input output module (DIOM). The DIOM sequences all accepted data and sends them to the readout module (ROM) for further storage and analysis via a 1 gigabit fiber-optic link. An abstract block diagram of this system can be seen in Figure 2 .

The cables connecting the FEAs to the DIOM are essentially the bottleneck in the data acquisition system. The data for each of the 16 FEEs that compose a quadrant of the DCH electronics are transmitted on 1/16 of the cable. Dead time occurs when the data volume generated per unit time by the large amount of accepted events in one FEE is greater than the carrying capacity of $1 / 16$ of the cable. These ratios are not adjustable; thus, if one channel is producing more data than $1 / 16$ of the cable can carry, and another FEE is producing little or no data, the system will experience dead time. The solution to this problem is either to increase the system's clocking speed, to enhance the cable capacity, or to redesign the system so that the amount of data sent through does not exceed the cable's carrying capacity. Advances in chip technology and availability of extra ROMs have made the second option 


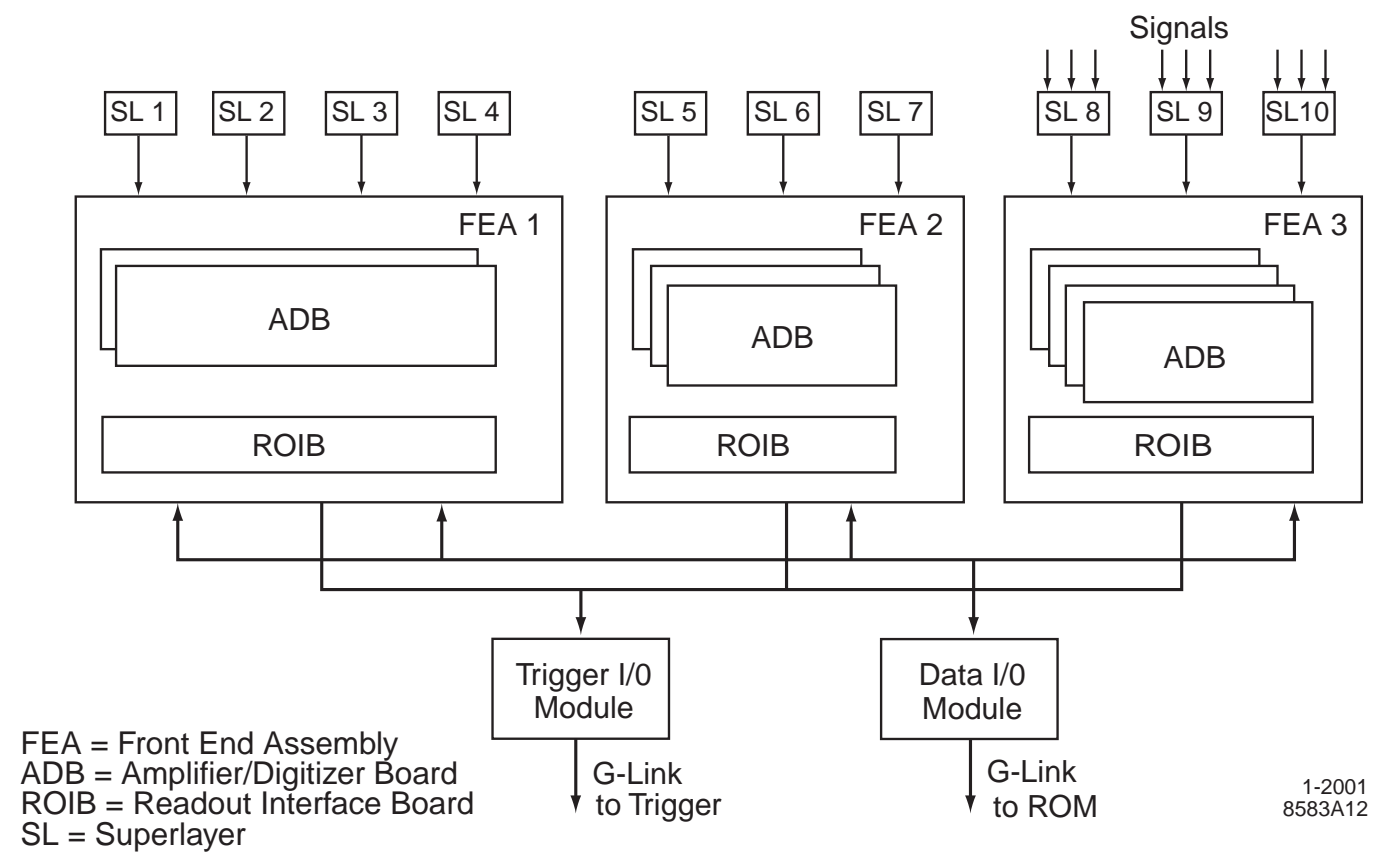

Figure 2: A block diagram of the DCH DAQ system. Taken from [2].

the most cost-effective and realistic.

The Phase II upgrade being implemented in the FEAs is the physical replacement of an older ROIB chip with a newer ball-grid array FPGA that uses increased memory and rewritable firmware to perform more tasks and lower the dataflow through the system. Pulse-height integration and pedestal removal functions that were previously performed in the ROM can now take place in the FEA. Thus, instead of sending a digitized, tagged waveform through the system, data are reduced to a single set of numbers that indicates the total deposited charge and location of trigger hits in the buffer. Whereas the initial DCH electronics setup produced 32 bytes of data per wire hit, the Phase II configuration will create a variable package size that averages to about 6.5 bytes per wire hit. Even at higher expected future luminosities, this change, along with respective alterations in data flow parallelism, will lower the dead times to less than $5 \%$, . 
In the first four years of BaBar's operation, most of the $4 \%$ dead time was caused by detector component malfunctions. Therefore, it is imperative that accepted changes in the BaBar detector are completely operational before they are implemented. The purpose of this paper is to explain the process by which Phase II changes in DCH ROIB boards were tested and to present and discuss the results of those tests.

\section{Methods and Materials}

\section{$3.1 \quad$ Overview}

For the DCH electronics upgrade, 81 new ROIB boards were fabricated to fill the 48 available spaces in the DCH FEAs. The 33 extra boards will be used to provide hardware for a DCH prototype and replacements for possible future failures. In order to make sure the upgraded DCH electronics are operational before their implementation, a series of tests can be performed on all functions and features of the new RoIBs. Real-time processes and final results of tests must also be catalogued in log files and databases for review and analysis.

Tests on the ROIBs are conducted using three computer-controlled test stands, some of which were used for tests of the original DCH FEAs and their components. On the component and calibration test stands, no modification is necessary for Phase II upgrade testing. The Drift Chamber Trigger (DCT) test stand was built after the initial construction of BaBar to provide a rigorous testing environment for all new BaBar hardware and software changes. The FEAs, test stands, and software were thoroughly tested beforehand to ensure that any failures in the testing process could be attributed to the ROIB boards themselves, rather than to testing apparatus and software. 
This allows for easy identification of badly manufactured boards.

On each day of testing, six new ROIBs are connected into six previously tested FEAs after a thorough visual inspection of each board. Next, the FEAs are attached one at a time in any order to the component test stand and the calibration test stand. For these stands, the board is tested by using a computer terminal to input commands into an IOC directly or via UNIX protocols. If the performance of each board in these two test stands is deemed acceptable, the six FEAs are then placed simultaneously in the DCT test stand and run as a group. Each stand conducts a variety of tests; their names and basic functions are listed in Table 1.

\subsection{Component Test Stand}

Also known as the vROM test stand, the component test stand is designed to check the functionality of specific FEA processes. The FEA is not physically situated in the test stand, but is plugged into a power supply and incorporated into the test stand hardware via a serial data cable. Fans placed on either side of the FEA keep temperatures within an acceptable operating range. For this stand, pulses of known duration and size are generated by discharging calibration circuits surrounding ELEFANT chips inside the FEA. Thus the signal input pins, normally the sources of incident pulses, are left unattached. A metal covering protects them from being accidentally bent or broken.

The individual components of the FEAs are checked by six separate tests in the VROM stand. The first, fifth, and sixth tests (featest 1, featest 5, and featest 6) are devoted to checking how an FEA and its components respond individually. Individual responses of registers and ELEFANTs are examined in featests 1 and 6 , while featest 5 checks buffer readout of individual ELEFANTs. 


\begin{tabular}{|c|c|c|}
\hline Test Stand & Test Name & Function of Test \\
\hline \multirow[t]{6}{*}{ Component } & Featest 1 & Checks response of individual registers \\
\hline & Featest 2 & Checks that ELEFANTs go into count mode correctly when prompted \\
\hline & Featest 3 & Makes sure that trigger hits read out as expected \\
\hline & Featest 4 & Tests pulse-height readout; Checks for proper signal gain \\
\hline & Featest 5 & Checks individual ELEFANT buffer readouts \\
\hline & Featest 6 & Calls each ELEFANT individually and checks for its response \\
\hline \multirow[t]{4}{*}{ Calibration } & Internal/External Gain (G) & Tests signal gain; similar to featest 4 \\
\hline & Noise Floor (NF) & Finds and examines pedestal height of every channel \\
\hline & Slew $(\mathrm{S})$ & Checks amplitude versus threshold crossing time of pulses \\
\hline & Internal/External Linearity (L) & Checks that time offsets between pulses are properly sent \\
\hline \multirow[t]{3}{*}{$\mathrm{DCT}$} & Trigger Pattern Calibration $(\mathrm{T})$ & Displays visually the agreement between raw data and TDC hits \\
\hline & Synch-Pulse (SP) & Checks buffer readout of four nearly simultaneous events \\
\hline & Long-Term (LT) & Looks for similarity of ELEFANT output for millions of events \\
\hline
\end{tabular}

Table 1: A summary of tests and their functions 
If the correct ELEFANT or register responds in an acceptable manner when called, the test is successful. Featests 2, 3, and 4 are tests that, when passed, will result in the return of identical output from all channels. Featest 2 tests whether or not all ELEFANTs can be brought into count mode; success in this test assures that the system's clocking is correctly configured and operational. Once proper clocking is assured, triggering and pulse-height portions of the FADC data can be monitored for proper readout in featests 3 and 4 , respectively. Differing output between any channels in these tests will result in a failure.

During the testing process, each featest is entered into the computer terminal individually, and then, if all pass, tests can then be repeated in sequence by using the command "featest 99". After approximately ten successful repetitions, testing can be considered complete.

\subsection{Calibration Test Stand}

Initially used to calibrate gain, triggering, and pedestal height for the original DCH FEAs, the calibration test stand is now used to check the functionality of the FEA as a whole. Unlike the component test stand, pulses are generated externally and fed from stand-based electronics into the FEA via the back-end signal inputs. Because of this, the FEA must be screwed tightly into place on a water-cooled structural frame resembling that of the DCH's. To reduce backgrounds in the tests, the FEA is left to cool down for fifteen to twenty minutes before testing.

Triggered test signals are read out from the front of the FEA through a serial-link cable and interpreted differently depending on the test. For the internal and external analog gain tests, a failure occurs when the gain of an outgoing signal from an FEA is different than expected. The noise floor test 
measures pedestal heights and compares them to reference values. The cause of a failure in this test is an excessively high pedestal, which results from noise in the test stand, increased background because of high temperatures, or because of improper wiring in new or old FEA components. Linearity tests check to see if time offsets corresponding to various known delay lengths between pulses are transferred properly through the FEA.

\subsection{DCT Test Stand}

After the FEAs have been run through the component and calibration test stands, they are sent in a group of six to the DCT test stand area. The test stand is an operating mockup of the BaBar drift chamber; it incorporates identical software and sections of the DCH hardware. For example, instead of running sixteen azimuthally symmetric electronics sections for data acquisition, the DCT test stand runs only two. It contains one DIOM and one trigger input output module (TIOM), whereas the drift chamber has four DIOMs and eight TIOMs. Because of the similarity between the test stand and the full DCH, new software for the DCH can be tested on the DCT system before being implemented in the detector. Parts can also be swapped directly out of the DCT stand into the DCH. The one main difference between the two setups besides size is signal source: as in the component test stand, signals are generated by calibration circuits inside the FEA itself.

Three tests are run by the DCT test stand. The trigger pattern calibration test attempts to match raw data trigger hits on groups of channels from the TIOM with subsequent trigger data read from ELEFANT buffers to the ROMs via the DIOM. BaBar DCH software visually plots incoming data from the two systems in an end-on view of the $\mathrm{DCH}$; if the test is successful, all raw trigger hits correspond to TDC hits in the same location. The synch-pulse test 
addresses a problem identified in some of the current ELEFANT ICs. When four events are read out as fast as possible in one ELEFANT buffer, clocking information and FADC data are often confused with one another. By sending in four quick pulses, the synch-pulse test determines whether or not this is also the case with the new boards. The long-term test runs ELEFANTs in count mode overnight and logs upwards of $2 \times 10^{8}$ events, allowing the reliability of the ELEFANTs to be tested very rigorously.

\subsection{Test Records}

In order to keep track of new boards and their performance history, various forms of documentation have been created. To record in-test processes, a log file is created in the ROM window where commands are executed and data is returned from the FEA. All successes and failures are documented in this file; unfortunately, one must search through many lines of code to find them. The advantage of log files is that they contain the exact messages the software returns upon finding a failed event. This allows easy access for troubleshooting.

To have an overview of the entire testing process on all boards, hardware and testing databases have been created. Directly after a test on an FEA is concluded, the FEA serial number, ROIB number, and test start and end time are recorded in the testing database, along with information on which tests, if any, the FEA failed. Comments concerning test results can also be recorded. This simple system allows boards to be tested in an efficient and organized manner. 


\section{Results and Discussion}

The purpose of testing the Phase II electronics upgrade is to identify badly manufactured boards and to uncover any noteworthy behavior in the new components before they are put into the DCH. Bad boards are sent back to the manufacturer, while unreliable ones are relegated to serving as backups for more dependable new units.

Out of the 81 new ROIB boards tested, 17 failures were documented in the testing database. However, the database was only marginally helpful in gauging how many problems actually occurred during the course of testing. In some cases, immediate troubleshooting of the test stands and FEAS after a failure solved the problem. For this situation, the test was recorded as successful in the database, despite the initial problems. Some events recorded in the database as failures were problems of the same nature as the ones just previously mentioned, only that the failed status was reported before troubleshooting solved the problem. Ultimately, log files for the tests were the best places to look for actual errors that occurred during the testing process. A short list of commonly failed tests and their reasons for failure can be seen in Table 2 .

The component test stand identified one operational problem that occurred in some of the FEA 1 ROIBs. During featest 3 on ROIB boards 20004738, 20004782, 20004798, 20004807, and 20004818, read and predicted values on a small number of trigger buffers did not agree with one another, resulting in failed tests. During the troubleshooting process, it was observed that the delay chips on all failing RoIBs were manufactured at different times than the delay chips on the rest of the boards. The discrepancy in delay caused by the different chips was reconciled by putting an extra jumper on all offending boards. After the addition of this new part, the boards proceeded to behave 


\begin{tabular}{|c|c|}
\hline Test Name & Reason for Failure \\
\hline Featest 3 & Triggers not in sync; differing delay chips \\
Featest 4 & Box not grounded; ambient noise \\
Internal and External Gain & Probable bad component on ADB \\
Noise Floor & Temperature-dependent background; improper ADB connection \\
Slew & Temperature-dependent background \\
Internal and External Linearity & Not fully understood; test stand-related \\
DCT tests & Network and database issues, delay chips, badly written data from ROIBs, \\
& lack of control over the DCT environment \\
Visual & various causes; errant soldering, etc. \\
\hline
\end{tabular}

Table 2: A short summary of failed tests and their reasons for failure 
normally. These 2-jumper ROIBs will be kept as backups for DCH FEAs when the Phase II upgrade is installed.

The remaining featests on the component test stand were relatively uneventful. Featests 1, 2, 5, and 6 had no failures at any point. Featest 4 failed intermittently. Over the course of testing 81 boards, 43 one-channel featest 4 failures occurred at a rate of approximately one error for every 2000 to 4000 channels tested, depending on the FEA type. The failures were the result of ungrounded FEAs; on the component test stand, signal input pins were left floating, which allowed baseline fluctuations in the signal from ambient noise. Such a fluctuation contributes to an incorrect signal gain and pulse-height readout.

The calibration test stand, while incurring some fairly frequent failure modes, did not uncover any abnormal ROIB behavior. A ubiquitously occurring error was reported by the noise floor test. Despite waiting fifteen to twenty minutes for temperature-related background to dissipate, the reported pedestal remained at unacceptable values. As boards flawlessly passed triggering and pulse-height tests on other test stands, the problem was attributed to noise in the test stand itself. Any noise floor test with less than ten errors was disregarded. Two occurrences of higher noise floor problems, 36 and 42, for boards 20004788 and 20004782, occurred when ADB boards were not properly mounted on their FEA. The slew test failed intermittently, but these problems were also temperature-related and disregarded if problems occurred on an isolated basis.

More non-ROIB related failures were also noted on the linearity and gain tests. Three out of twelve gain tests on FEA \#127 failed only at the highest settings, most likely due to a malfunctioning component on an ADB board. One board failed all gain tests for four channels and all linearity tests for all 
channels. The offending FEA had passed related tests on other stands, so the problem was attributed to the test stand once again. The cause of failure has yet to be specifically determined. Later calibration tests on the same ROIB were completely successful.

The DCT test stand had more failures per test than either of the other two test stands, some of which were attributable to bad readout input boards. A chart of all ROIB failures can be found in Table 3. Board 20004764 was found to be improperly communicating its synchronization status: although it was locked with the system's clocking times, it was reporting that it was out of synchronization. This problem displayed itself as damage on the trigger pattern calibration test. Along with a delay chip problem, board 20004798 occasionally wrote tag bits and header bits in incorrect locations, causing a similar kind of failure. These problems could be the result of bad soldering in the ROIB board itself. These two ROIBs, as well as another board (20004783) with a massive shorting solder found during visual tests, are the only completely non-functioning boards.

The rest of the DCT failures were not ROIB-related. The complexity of the DCT test is one reason so many failures were encountered. Rather than running independently from other systems, the DCT stand has to communicate with ROMs from various locations, which means that all ROMs, databases, and networks must be operational in order for testing to proceed successfully. The DCT stand is also used for a variety of purposes, including trigger and software experimentation. If non-ROIB-related work on the DCH mockup has altered any DCT operating procedures or environments, Phase II upgrade tests will return failures. It is also worth noting that some of the failures on this test stand are recurrences of problems found on previous test stands. For example, the delay chip problem first identified on the VROM stand caused 


\begin{tabular}{|c|c|c|c|c|c|c|c|c|c|c|c|c|c|c|}
\hline ROIB \# & Visual & $\mathrm{F} 1$ & $\mathrm{~F} 2$ & F3 & $\mathrm{F} 4$ & F5 & $\mathrm{F} 6$ & $\mathrm{G}$ & $\mathrm{NF}$ & $\mathrm{S}$ & $\mathrm{L}$ & $\mathrm{T}$ & $\mathrm{SP}$ & LT \\
\hline 20004738 & . & . & . & $x$ & . & . & • & . & . & . & . & . & . & . \\
\hline 20004764 & . & . & . & & • & • & . & . & & . & . & $x$ & $x$ & - \\
\hline 20004782 & . & . & . & $\times$ & . & . & . & . & . & . & . & . & . & . \\
\hline 20004783 & $x$ & - & - & - & - & - & - & - & - & - & - & - & - & - \\
\hline 20004798 & . & . & . & $\times$ & . & . & . & . & 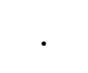 & . & . & $\times$ & $x$ & - \\
\hline 20004807 & . & . & . & $\times$ & . & . & · & . & & . & . & . & . & . \\
\hline 20004812 & $\cdot$ & . & . & $\times$ & . & . & . & . & $\cdot$ & . & . & . & . & . \\
\hline 20004818 & . & . & & $\times$ & . & . & 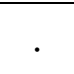 & . & & . & . & . & . & . \\
\hline
\end{tabular}

Table 3: Bad or unusually behaving boards and their failed tests. Symbol key: ·, passed test; $\times$, failed test; -, not tested. Test name abbreviations: F, Featest; G, Internal/External Gain; NF, Noise Floor; S, Slew; L, Internal/External Linearity; T, Trigger Pattern Calibration; SP, Sync-Pulse; LT, Long-Term. 
data to be improperly written from board 20004812 in the DCT stand.

To summarize overall ROIB performance the results from all three test stands and visual testing should be viewed as a whole. Three boards were completely non-functional, and five others displayed slightly different but fixable delay behaviors. Nine boards need to be tested more thoroughly, either because they missed certain test stands or because they experienced some unpredictable and possibly anomalous failures during the testing process. Four boards have yet to be assembled or tested.

\section{Conclusion}

Overall, the functionality and short-term reliability of the ROIB boards has been proven through the course of testing, with few exceptions. Out of 81 boards, the three completely non-functional boards will likely be sent back to the manufacturer for replacement. The five FEA 1 boards with delay problems only will be used as backups for more reliable failure-free boards. Thus, 73 completely functional and reliable boards remain to be used for various purposes. Six ROIBs will be installed permanently on the DCT test stand to be used for developing or troubleshooting triggering and software problems in the DCH. Two will be integrated into the Proto-2 drift chamber prototype, which is located near the DCT test stand. The remaining boards will either be installed into the 48 available ROIB slots on the front end of the BaBar drift chamber electronics or will serve as spares for failed boards in the DAQ system. In the event of massive drift chamber electronics failures, many replacements will be ready and available for installation to restart the experiment as soon as possible.

Besides checking for functionality of ROIB boards in the Phase II upgrade, hardware testing has also served other important purposes. First, it has 
allowed the BaBar group to observe the performance of new ROIBs without having to use them in the drift chamber itself. This experience with the upgrade allows for easier and quicker troubleshooting of problems during drift chamber operation. In addition, testing has identified important problems in other aspects of the electronics. Software and non-ROIB related hardware problems, such as incorrectly connected or malfunctioning ADB boards, were noticed and will be fixed before implementing the upgrade. Without testing the ROIBs, these problems could have gone unnoticed, causing failures in the drift chamber and interruptions in data-taking.

Future work will include longer-term testing of all FEA components. When it is certain that the properly tested Phase II upgrade can operate reliably in the BaBar detector, the changes will be implemented. In its operation, the upgrade will reduce dataflow through the system, significantly decreasing dead time without sacrificing detector reliability.

\section{Acknowledgements}

I would like to acknowledge Karl Bouldin and Mike Kelsey for their help in learning about and operating the DCH electronics system and test stands, and for solving all those problems that were beyond my expertise. I also want to thank Jochen Dingfelder, Vera Luth, and the rest of SLAC Research Group C for their help and guidance during the course of the project, and the Department of Energy, Office of Science for making my internship possible.

\section{References}

[1] DCH DAQ upgrade Working group, Jessop et al. "Proposal to Upgrade the BaBar Drift Chamber Electronics." February 6, 2004. 
[2] BABAR Collaboration, B. Aubert et al. "The BABAR detector," in Nuclear Instruments and Methods in Physics Research A, Vol. 479, pp. 1-116. 